Validation of the Use of the NMR MOUSE for Detection of Inhomogeneities in DC745 at Production

S. Chinn, A. Sawvel, R. Maxwell

November 16, 2004 
This document was prepared as an account of work sponsored by an agency of the United States Government. Neither the United States Government nor the University of California nor any of their employees, makes any warranty, express or implied, or assumes any legal liability or responsibility for the accuracy, completeness, or usefulness of any information, apparatus, product, or process disclosed, or represents that its use would not infringe privately owned rights. Reference herein to any specific commercial product, process, or service by trade name, trademark, manufacturer, or otherwise, does not necessarily constitute or imply its endorsement, recommendation, or favoring by the United States Government or the University of California. The views and opinions of authors expressed herein do not necessarily state or reflect those of the United States Government or the University of California, and shall not be used for advertising or product endorsement purposes.

This work was performed under the auspices of the U.S. Department of Energy by University of California, Lawrence Livermore National Laboratory under Contract W-7405-Eng-48. 


\title{
Validation of the Use of the NMR MOUSE for Detection of Inhomogeneities in DC745 at Production
}

\author{
Sarah Chinn, April Sawvel, and Robert Maxwell \\ Lawrence Livermore National Laboratory \\ Chemistry and Material Science Directorate
}

Executive Summary: In an effort to develop a QA/QC protocol to be used in the development of new pads, we are employing a tabletop unilateral NMR relaxometer called the NMR MOUSE (MObile Universal Surface Explorer). Model materials of varying crosslink density first demonstrated the applicability of the method. Analysis of deformed pads returned from service has been shown to be clearly distinguishable by the NMR MOUSE. Finally, we have quantified the variables associated with taking the relevant measurements and believe that the NMR MOUSE is a viable production and surveillance tool for screening of future DC745 parts.

Problem Overview: Approximately 20\% of outer pressure pad (OPP, part number 422431) surveillance returns have exhibited local areas of permanent compression and/or rib distortion. Numerous lab tests have been performed to try to reproduce such compression, but these tests have not succeeded in finding any conditions that lead to such damage. It was therefore concluded that the problem lies in the production side that is, there is a localized, uneven distribution of crosslink densities throughout the pad, leading to a lower resistance to load and a resulting damage to these parts of the pads.

The NMR MOUSE: Originally commercialized for the tire industry as an analytical tool for determining defect sites, the MOUSE is a transportable, inexpensive and easy to use system based on the principles of nuclear magnetic resonance. The MOUSE is currently used to determine mechanical properties of polymers, fat content of dairy products, and is even used in the preservation of artifacts such as historical papers and ancient stone ruins. With the appropriate choice of experimental parameters, the MOUSE is an excellent QA/QC tool for examining the OPPs before the pad is put into service. We have determined that adequate spatial resolution exists to identify areas of incomplete mixing in the final polymer pad, even if no visible differences are present. The ease of use and low cost compared with traditional NMR spectrometers is ideal for implementation into a polymer production plant.

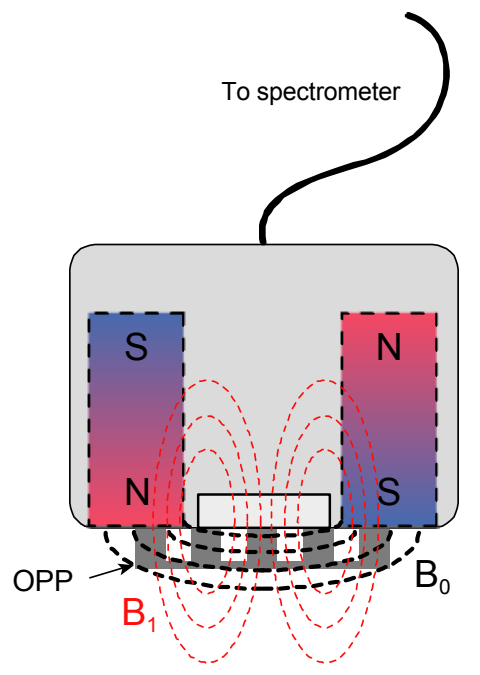

Figure 1. Schematic of MOUSE magnet unit, showing the $\mathrm{B}_{0}$ (black dashed) and $\mathrm{B}_{1}$ (red dashed) magnetic field lines and the DC745 sample (dark grey). 


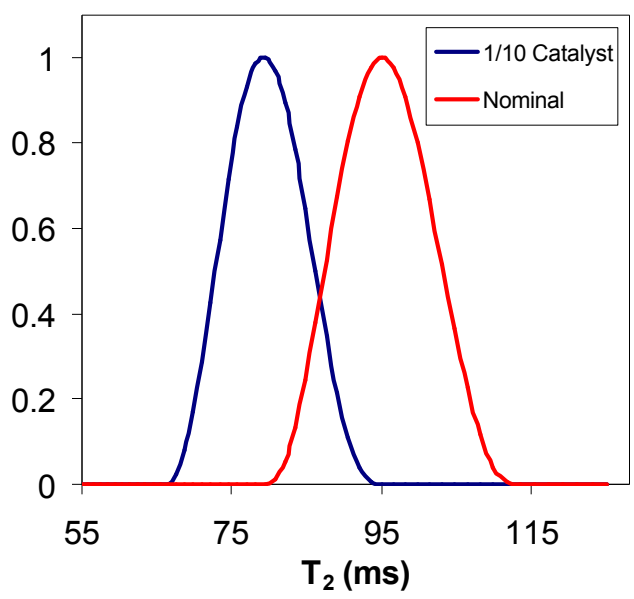

Figure 2. $\mathrm{T}_{2}$ distribution curve for nominal and $1 / 10$ catalyst DC745.
The MOUSE uses the same principles of traditional NMR spectroscopy, but since only relaxation information is obtained, the normal high resolution that is typically required for complete structural analysis is not needed here. In traditional NMR spectroscopy, a sample is irradiated with a rf pulse and the response of the nuclei are observed in the time-domain free induction decay (FID), after which a Fourier transform produces the frequency-domain spectrum. In the case of relaxometry, the initial rf pulse is applied followed by a separate train of pulses that refocuses the magnetization

into an "echo". The intensity of this echo decays in time with a time constant $\mathrm{T}_{2}$ as the magnetization is transferred to neighboring spins. Because a Fourier transform is never used and high frequency resolution is not needed, highly homogeneous static $\left(\mathrm{B}_{0}\right)$ or excitation $\left(\mathrm{B}_{1}\right)$ magnetic fields are also not required.

The NMR MOUSE consists of a computer, tabletop spectrometer console and preamplifier, and a magnet unit. The magnet unit consists of two permanent magnets with anti-parallel magnetization producing a $\mathrm{B}_{0}$ field parallel to the surface of the unit, as shown in Figure 1. $\mathrm{B}_{1}$ irradiation is applied with a surface $\mathrm{rf}$ coil in the center producing a smaller magnetic field perpendicular to the surface. The MOUSE can be held by hand or by a robotic controller and scanned systematically over the entire surface of the OPP. The spatial resolution is approximately $1.5 \mathrm{~cm}^{2}$, about the width of two ribs, which is of comparable size to the deformed areas of the pads.

Results: NMR spectroscopy has been used to determine relative crosslink densities in polymers for some time. The relaxation properties obtained through high resolution NMR experiments has been shown to correlate with various mechanical properties of polymers. For example, the $\mathrm{T}_{2}$, or spin-spin, relaxation time is proportional to $1 /(\mathrm{CLD})^{2}$. However, it is not possible to put an entire OPP in the NMR spectrometer for analysis. We have chosen an alternative method to obtain relaxation measurements of an intact OPP. The first step in the implementation of the MOUSE to the OPP issue was to verify that the sensitivity of the MOUSE to the expected $\mathrm{T}_{2}$ differences is great enough and that the data obtained

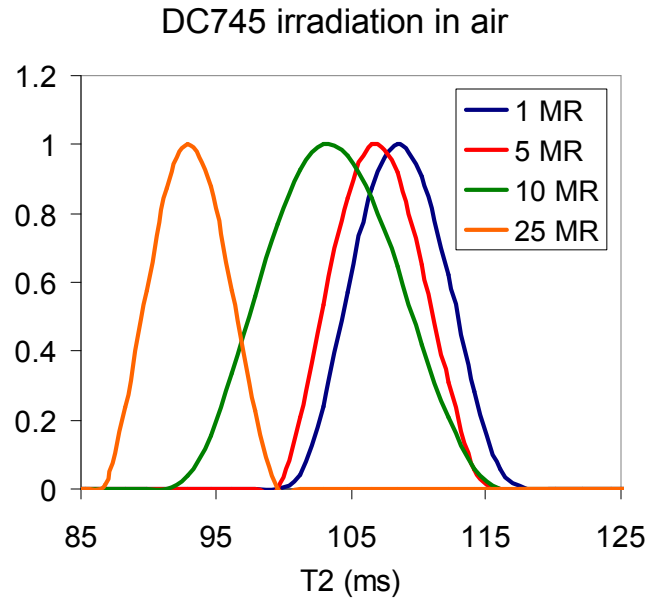

Figure 3. Effects of Co-60 irradiation on DC745. 


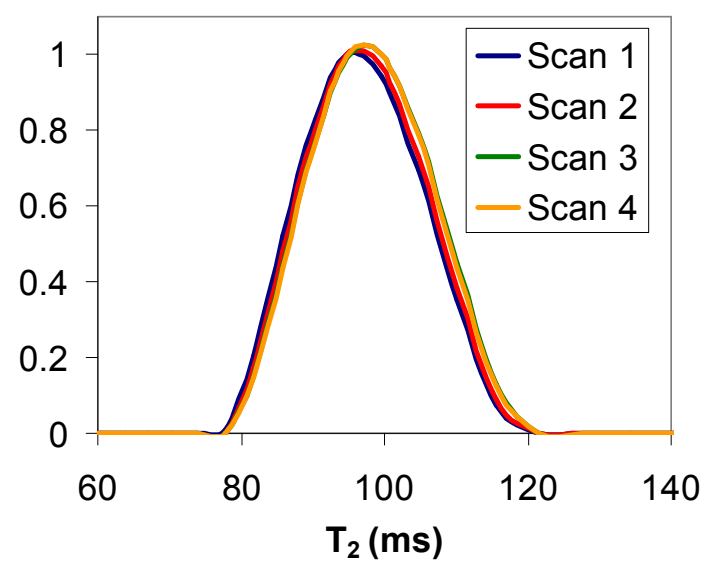

Figure 4. Results of repeated scans on the same spot of a DC745 pad.

been observed in NMR observables such as $T_{2}$ relaxation times and residual dipolar couplings. We have observed trends in $\mathrm{T}_{2}$ times as a function of radiation dose by high resolution NMR, namely that the $\mathrm{T}_{2}$ time decreases with increasing dose. These trends were confirmed using the NMR MOUSE, with the data shown in Figure 3.

In running routine $T_{2}$ measurements with the MOUSE, there are a few experimental parameters that must be set correctly in order to obtain accurate results. To obtain optimal resolution in $\mathrm{T}_{2}$ distribution a signal to noise ratio of at least 50:1 is desirable, requiring at least a few hundred scans. Additionally, the receiver gain of the spectrometer must not be set too high, as clipping of the first part of the signal with a resulting inaccurate determination of $\mathrm{T}_{2}$ time is possible.

The reproducibility of the MOUSE results is important in the QA/QC process. Numerous tests were performed to determine that the same $T_{2}$ values were obtained from the same spot on a plain, non-ribbed DC745 pad. Figure 4 was obtained with a high signal to noise ratio to yield extremely reproducible results.

As the outer pressure pads have a complex rib design, it was necessary to determine the effect of the sharp edges on the MOUSE results. We tested these effects by acquiring data at varying distances from the edge of a plain, nonribbed DC745 pad. Figure 5 shows that as long as the pad is covering $1 / 2$ of the detection volume of the surface coil the $T_{2}$ times did not change appreciably.

The final set of tests was on an actual damaged OPP. Figure 6 shows the differences in $\mathrm{T}_{2}$

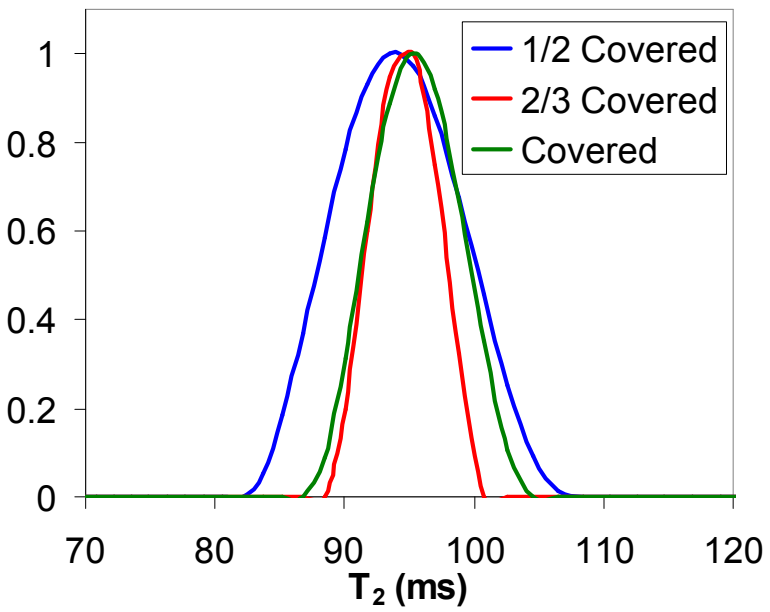

Figure 5. Edge effects on a plain DC745 pad. 

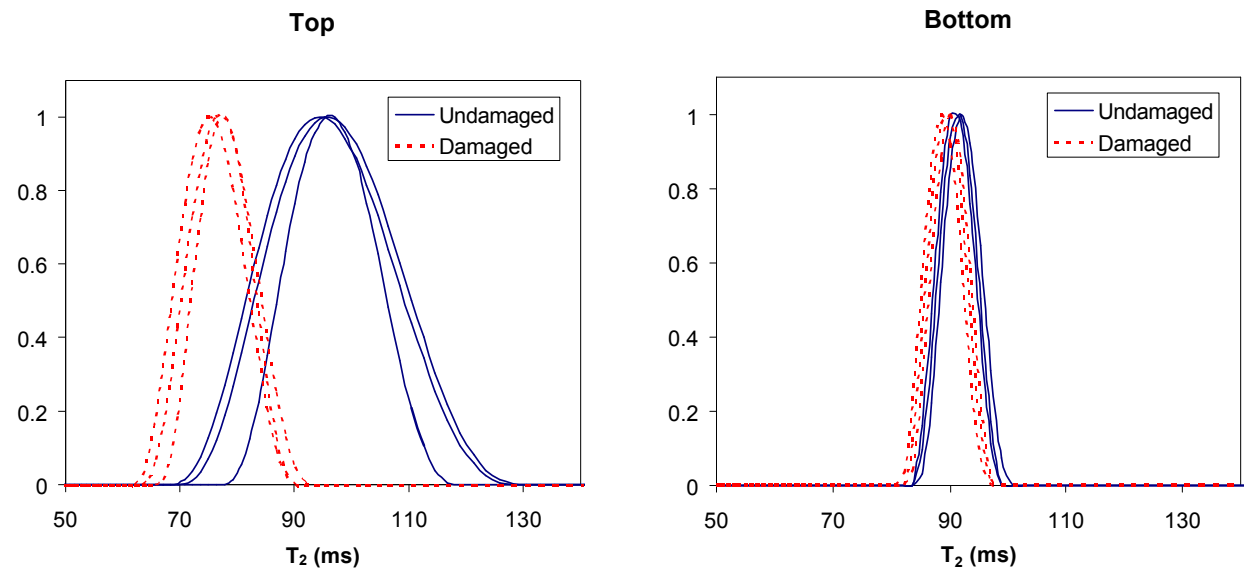

Figure 6. Damaged and undamaged spots from damaged DC745 outer pressure pad.

relaxation time between a damaged and undamaged section of a real pad. Since the surface coil on the MOUSE only penetrates a small distance into the polymer pad, scans were run on both the top and bottom of the pad. If the defect is only in one portion of the pad and not present in the entire thickness of the pad, the changes in $T_{2}$ might not be observable if only one side was scanned. In Figure 6, distinct changes are more observable in the top section of the polymer, indicating that the defect does not in fact go through the entire depth of the pad. 